P. Gastmeier

M. M. Hoeper

M. Stoll

H. A. Adams

\title{
Der Notfallplan des Krankenhauses bei allgemeingefährlichen Infektionskrankheiten
}

Hospital emergency plan for the management of patients with highly contagious diseases

Abstract Patients with imported highly contagious diseases like viral hemorrhagic fever (VHF), pneumonic plague or anthrax require special preventive measures and strict isolation. In addition, new emerging infections like severe acute respiratory syndrome (SARS) and epidemic influenza also have to be taken into account. At present five high security infectious disease units for patient care are available in Germany. Due to the relatively nonspecific symptoms, a patient with one of these infections may also be admitted to any other hospital. Therefore each hospital should develop a concept for the management and control of these lifethreatening highly contagious diseases. The present article describes the current emergency plan developed for the management of patients with highly contagious diseases at Hannover Medical School.

Key words infections contagious diseases emergency plan
Zusammenfassung Patienten mit importierten hochkontagiösen Erkrankungen wie virusbedingtes hämorrhagisches Fieber (VHF), Lungenpest oder Lungenmilzbrand erfordern spezielle Präventionsmaßnahmen und strikte Isolation. Darüber hinaus müssen neue Infektionskrankheiten wie Severe Acute Respiratory Syndrome (SARS) und epidemische Influenza beachtet werden. Zur Zeit gibt es in Deutschland fünf überregionale Kompetenzzentren zur Behandlung dieser Patienten. Wegen der relativ unspezifischen Symptome kann ein Patient mit einer dieser Infektionen aber auch in ein anderes Krankenhaus eingewiesen werden. Deshalb soll jedes Krankenhaus ein Konzept für die Behandlung und die Kontrolle dieser lebensbedrohlichen hoch-kontagiösen Erkrankungen entwickeln. Der vorliegende Artikel beschreibt den aktuellen Notfallplan, der an der Medizinischen Hochschule Hannover für die Behandlung dieser Patienten entwickelt wurde.

\section{Schlüsselwörter}

Infektionen - hochkontagiös Notfallplan

Notfall- und Katastrophenmedizin

Medizinische Hochschule Hannover 


\section{Einleitung}

Allgemeingefährliche Infektionskrankheiten können im Zusammenhang mit Patientenreisen in Endemiegebiete $\mathrm{zu}$ Problemen in unseren Krankenhäusern führen. Vor allem aber nach dem Herbst 2001 ist auch die Gefahr von bioterroristischen Angriffen verstärkt in das Bewusstsein gerückt. Darüber hinaus können neue, bisher nicht bekannte Infektionserreger auftreten und $\mathrm{zu}$ Infektionsausbrüchen in Krankenhäusern führen, wie uns das Beispiel des Severe Acute Respiratory Syndrome (SARS) gezeigt hat - und wie wir es möglicherweise für die schwere pandemische Influenza fürchten müssen.

Glücklicherweise erleben die meisten Ärzte während ihres Beruflebens keinen Fall allgemeingefährlicher Infektionskrankheiten oder höchstens einmal einen Verdachtsfall. Deshalb ist das Wissen über die in solchen Fällen notwendigen Schritte wenig verbreitet. Vor allem wird immer wieder beobachtet, dass wegen der Unsicherheiten über die Ausbreitung der Erreger völlig überzogene Maßnahmen angeordnet werden und dadurch unnötige Panik erzeugt wird. Deshalb ist es sinnvoll, den Verdachtsfall einer allgemeingefährlichen Infektionskrankheit für das eigene Krankenhaus einmal „durchzuspielen“ und die Ergebnisse in einem Notfallplan festzulegen. Einer entsprechenden Koordinationsgruppe sollen mindestens ein Vertreter der Krankenhausleitung, der Krankenhaushygieniker, der Infektiologe, der Leiter der Notfallmedizin/Rettungsstelle und der Mikrobiologe angehören.

Bei einem Verdachtsfall auf eine allgemeingefährliche Infektionskrankheit muss eine vorab bestimmte Führungsstruktur aktiviert werden. Wichtige Aufgaben sind die Koordination der Patientenbehandlung, der Schutz der potentiell exponierten Personen und die Kommunikation mit den übrigen Krankenhausabteilungen und nach außen. Der folgende Artikel beschreibt exemplarisch die Überlegungen, die dem entsprechenden Notfallplan der Medizinischen Hochschule Hannover zugrunde liegen.

\section{Allgemeingefährliche Infektionskrankheiten und ihre Übertragungswege}

Allgemein werden drei mögliche Wege der Infektionsausbreitung unterschieden:

- der Kontakt (direkt oder indirekt über kontaminierte Hände oder Instrumente),

- die Tröpfcheninfektion und

- die Ausbreitung über Aerosole.

Große Erreger sind in der Regel an Tröpfchen gebunden und sedimentieren gemeinsam mit den
Tab. 1 Sedimentationsgeschwindigkeit in Abhängigkeit von der Erregergröße nach Papineni [1]

\begin{tabular}{llllll}
\hline Erregergröße in $\mu \mathrm{m}$ & 0,5 & 1 & 3 & 10 & 100 \\
Sedimentationsdauer & $41 \mathrm{~h}$ & $12 \mathrm{~h}$ & $1,5 \mathrm{~h}$ & $8,2 \mathrm{~min}$ & $5,8 \mathrm{~s}$ \\
\hline
\end{tabular}

Tab. 2 Zuordnung von Infektionen zu den verschiedenen Übertragungswegen [2]

\begin{tabular}{|c|c|c|c|}
\hline Beispiele & $\begin{array}{l}\text { Kontakt- } \\
\text { übertragung }\end{array}$ & $\begin{array}{l}\text { Tröpfchen- } \\
\text { infektion }\end{array}$ & $\begin{array}{l}\text { Übertragung } \\
\text { durch Aerosol }\end{array}$ \\
\hline $\begin{array}{l}\text { Häufige } \\
\text { Infektions- } \\
\text { erkrankungen }\end{array}$ & $\begin{array}{l}\text { Infektiöse } \\
\text { Durchfall- } \\
\text { erkrankungen } \\
\text { Clostridium- } \\
\text { difficile-Enteritis } \\
\text { Infektionen mit } \\
\text { multiresistenten } \\
\text { Erregern (MRSA, } \\
\text { VRE, ESBL) }\end{array}$ & $\begin{array}{l}\text { Meningokokken- } \\
\text { Meningitis } \\
\text { Pneumokokken- } \\
\text { Pneumonie } \\
\text { Pertussis } \\
\text { Diphtherie } \\
\text { Influenza } \\
\text { Mumps } \\
\text { Röteln }\end{array}$ & $\begin{array}{l}\text { Tuberkulose } \\
\text { Varizellen } \\
\text { Masern }\end{array}$ \\
\hline $\begin{array}{l}\text { Allgemein- } \\
\text { gefährliche } \\
\text { Infektions- } \\
\text { erkrankungen }\end{array}$ & $\begin{array}{l}\text { Virusbedingtes } \\
\text { hämorrhagisches } \\
\text { Fieber } \\
\text { Lungenmilzbrand }\end{array}$ & $\begin{array}{l}\text { Aviäre Influenza } \\
\text { SARS } \\
\text { Lungenpest }\end{array}$ & Pocken \\
\hline
\end{tabular}

MRSA Methicillin-Resistenter Staphylococcus Aureus; VRE Vancomycin-Resistente Enterkokken; ESBL Extended Spectrum Beta-Laktamasen; SARS Severe Acute Respiratory Syndrome

Tröpfchen relativ schnell im Umkreis der Infektionsquelle. Kleinere Erreger können sich auch frei in der Luft bewegen und haben somit verlängerte Sedimentationszeiten. Papineni und Rosenthal [1] haben die Sedimentationsdauer in Abhängigkeit von der Erregergröße gemessen (Tab. 1).

Dementsprechend verbreiten sich große, an Tröpfchen gebundene Erreger $(>5 \mu \mathrm{m})$ primär nur in der direkten Umgebung der Infektionsquelle (ca. $1 \mathrm{~m}$ Umkreis), während sehr kleine Erreger $(<5 \mu \mathrm{m})$ auch in großer Entfernung von der Infektionsquelle im Raum nachgewiesen werden können. Auf dieser Basis ist es möglich, Infektionserreger hinsichtlich des Übertragungsweges in vorrangig durch Kontakt übertragene Erreger, gebunden an Tröpfchen verbreitete Erreger und in solche einzuteilen, bei denen eine Ausbreitung über Aerosol möglich ist (Tab. 2).

$\mathrm{Zu}$ den lebensbedrohlichen hochkontagiösen Infektionskrankheiten gehören die virusbedingten hämorrhagische Fieber (VHF) wie Lassa, Ebola, KrimKongo-Fieber, südamerikanisches hämorrhagisches Fieber und durch Hantavirus bedingtes Fieber. Sie sind weltweit selten. Aufgrund des Fehlens einer spezifischen Therapie und einer hohen Letalität generieren sie allerdings Angst und Unsicherheit. Die Übertragung von VHF geschieht hauptsächlich durch direkten Kontakt und Tröpfchen, eine Übertragung durch Aerosole kann aber zur Zeit nicht ausgeschlossen werden [3]. 
Obwohl in manchen Influenza-Ausbrüchen die Übertragung über Aerosol angenommen wird, ist die Tröpfchen-assoziierte Transmission der häufigste Weg der Übertragung der Influenza [2]. Aufgrund der schnellen Ausbreitung der SARS-Fälle in Krankenhäusern im Jahr 2003 und ihrer hohen Letalität ist SARS ebenso im Notfallplan zu erfassen wie das Auftreten einer schweren pandemischen Influenza.

Im Zusammenhang mit biologischen Kampfstoffen sind insbesondere Anthrax, Botulismus, Lungenpest, Pocken und Tularämie relevant.

\section{Verdachtsmoment - Verdachtsfall - gesicherte Diagnose}

Der Notfallplan muss zwischen Verdachtsmomenten, Verdachtsfällen und Patienten mit einer gesicherten Diagnose einer allgemeingefährlichen Infektionskrankheit unterscheiden (Tab. 3).

Schon bei einem Verdachtsmoment soll ein Infektiologe konsultiert werden. Zu diesem Zeitpunkt sollen bereits die in der folgenden Checkliste aufgeführten Informationen durch den zuerst kontaktierten Arzt erhoben worden sein (Tab. 4).

Bereits beim Auftreten der ersten Verdachtsmomente sind entsprechende Schutzmaßnahmen für das Personal vorzunehmen. Dazu ist der unverzügli-

Tab. 3 Übersicht über Verdachtsmomente, Verdachtsfälle und den Status eines Patienten mit gesicherter Diagnose

\begin{tabular}{ll}
\hline Verdachtsmoment & $\begin{array}{l}\text { Situationen, in denen nach Auftreten von Sympto- } \\
\text { men oder einer definierten Expositionsanamnese } \\
\text { noch keine abschließende Risikokategorisierung als } \\
\text { Krankheitsverdacht im Sinne der fallspezifischen } \\
\text { Definitionen möglich ist } \\
\text { Krankheitsverdacht im Sinne der fallspezifischen } \\
\text { Definitionen }\end{array}$ \\
Patienten mit & $\begin{array}{l}\text { Nur wenn der Patient nicht verlegungsfähig ist } \\
\text { oder das Kompetenzzentrum keine Patienten mehr } \\
\text { aufnehmen kann }\end{array}$ \\
\hline
\end{tabular}

Tab. 4 Checkliste 1 - Notwendige Informationen beim ersten Kontakt mit dem Patienten

\footnotetext{
Welche Erkrankung wird vermutet?

- Wie viele Personen sind betroffen (Geschlecht, Alter)?

- In welcher Sprache ist eine Verständigung möglich?

- Bestehen Symptome? (Welche? - seit wann? - stabile Vitalparameter?)

- Risikoanamnese:

- Nachweislich exponiert?

- Nachweislich aus Hochrisikogebiet-/Endemiegebiet?

- Anderweitige Vermutung einer Exposition? Worauf gestützt?

- Wurde der Patient bereits von einem Arzt gesehen und untersucht?

- Wurden bereits spezifische Maßnahmen eingeleitet? - wenn ja, welche?
}

Tab. 5 Inhalt des Infektionsschutzsets

1 feinfiltrierende Partikelmaske (FFP-3) mit Ausatemventil (nicht für Patienten geeignet)

- 1 Arbeitsschutzbrille mit seitlichem Spritzschutz

- 1 OP-Kopfhaube

- 1 flüssigkeitsdichter Overall (ersatzweise Plastikschürze und hinten schließender Stoffkittel mit langen Ärmeln)

- 2 Einmal-Plastiküberschuhe

- 1 Paar lange Schutzhandschuhe

- 2 Pakete unsteriler Handschuhe (groß und mittelgroß)

Tab. 6 Falldefinition "Verdacht auf VHF“ [3]

- Patient mit Fieber $\left(\geq 38^{\circ} \mathrm{C}\right.$ axillär), der sich weniger als 3 Wochen vor Auftreten der Symptome in einer endemischen oder epidemischen Zone für VHF aufgehalten hat, oder den Körpersekreten einer Person oder eines Tieres mit VHF ausgesetzt war, für den keine andere Diagnose etabliert werden konnte.

- Patient mit Fieber und hämorrhagischer Diathese, der sich weniger als 3 Wochen vor Auftreten der Symptome in einer intertropischen Region Afrikas, in Lateinamerika oder Osteuropa aufgehalten hat, insbesondere, wenn in der betroffenen Region eine Epidemie mit ungeklärten Todesfällen im Gange ist.

Tab. 7 Falldefinition Verdacht auf Severe Acute Respiratory Syndrome (SARS) [RKI]

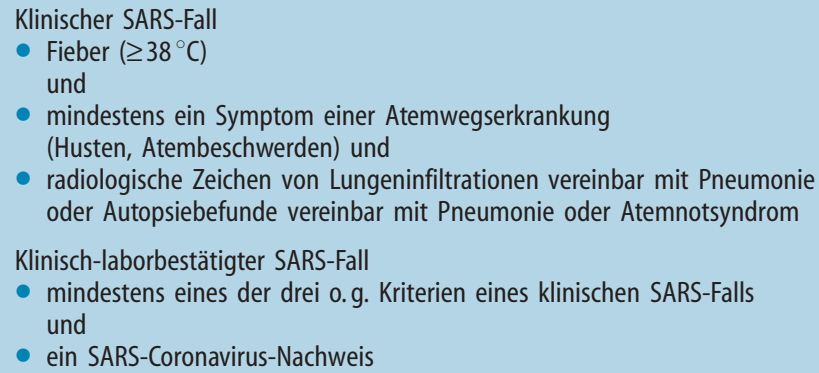

che Zugriff auf entsprechende Notfallsets erforderlich, die im Aufnahmebereich vorzuhalten sind (Tab. 5).

Anhand geeigneter Falldefinitionen wird der Infektiologe auf der Basis der vorliegenden Befunde sowie der anamnestischen und epidemiologischen Informationen entscheiden, ob dieser Patient als Verdachtsfall anzusehen ist. Im Folgenden sind beispielhafte Falldefinitionen für den Verdacht auf VHF, SARS und schwere pandemische Influenza aufgeführt (Tab. 6-8).

Die Information zu den aktuellen Endemiegebieten müssen den entsprechenden Internetseiten der WHO (www.who.int) entnommen werden, bezüglich der Falldefinitionen für andere allgemeingefährliche Infektionen verweisen wir auf die Homepage des Robert-Koch-Instituts (www.rki.de). 
Tab. 8 Falldefinition Influenzavirus A/H5 [Stand RKI 30. 03. 2006]

$\begin{array}{ll}\text { - Verdachtsfall: } & \begin{array}{l}\text { Erfülltes klinisches Bild, ohne Nachweis einer anderen } \\ \text { Ursache, die es vollständig erklärt, und mit epidemio- } \\ \text { logischer Exposition }\end{array} \\ \begin{array}{ll}\text { - Wahrscheinlicher } & \text { Verdachtsfall mit einem positiven labordiagnostischen } \\ \text { Fall: } & \text { Nachweis von A/H5 ohne Bestätigung durch ein Re- } \\ & \text { ferenzlabor }\end{array} \\ \begin{array}{ll}\text { - Bestätigter Fall: } & \text { Wahrscheinlicher Fall mit labordiagnostischem Nach- } \\ & \text { weis von A/H5, der durch ein Referenzlabor bestätigt } \\ & \text { wurde }\end{array}\end{array}$

Klinisches Bild = Erkrankung mit Vorliegen aller drei folgenden Kriterien: Fieber, akuter Krankheitsbeginn, Husten oder Dyspnoe oder Tod durch unklare akute respiratorische Erkrankung

Epidemiologische Exposition: Epidemiologische Exposition, definiert als mindestens eine der drei folgenden Expositionen innerhalb von 7 Tagen vor Erkrankungsbeginn:

(A) Kontakt mit Tieren oder deren Ausscheidungen bzw. Aufenthalt in einem Gebiet mit

laborbestätigten hochpathogenen aviären Influenzaviren

(B) Menschlicher Kontakt

(C) Laborexposition

\section{Konzentration der Behandlung in überregionalen Zentren}

Zur Sicherstellung der Behandlung von lebensbedrohlichen hochkontagiösen Infektionskrankheiten in Deutschland ist eine Konzentration auf wenige überregionale Kompetenzzentren vorgesehen (Abb. 1) [4]. Die Kompetenzzentren verfügen über Personen mit besonderem Fachwissen sowie die logistischen Voraussetzungen für die Behandlung entsprechender Patienten.

Sobald die Falldefinition einer allgemeingefährlichen Infektionskrankheit erfüllt ist, ist mit dem nächstgelegenen Kompetenzzentrum Kontakt aufzunehmen. Ziel ist, den Patienten so schnell wie möglich in das Zentrum zu transportieren. Dazu wird das abgebende Krankenhaus vom Kompetenzzentrum beraten. Weiter ist das zuständige Gesundheitsamt umgehend zu informieren.

\section{Ersatzweise Notfallbehandllung vor Ort}

Falls eine kurzfristige Übernahme durch das Kompetenzzentrum nicht möglich oder der Patient nicht transportfähig ist, muss er im erstaufnehmenden Krankenhaus verbleiben und dort behandelt werden. Ungerichtete Sekundärtransporte sind zu vermeiden. Im Notfallplan ist für diesen Fall eine besondere Behandlungszone auszuweisen, in der der Patient sowohl suffizient isoliert wie auch behandelt werden kann:

- Die besondere Behandlungszone soll von außen zugänglich und von den übrigen Klinikbereichen gut abgegrenzt sein.
- Reine und unreine Ver- und Entsorgungswege sind so gut wie möglich zu trennen.

- Die Belüftung muss zwingend vom allgemeinen Belüftungssystem getrennt sein; eine Unterdruckhaltung ist wünschenswert, aber nicht zwingend erforderlich.

- In der besonderen Behandlungszone ist eine intensivmedizinische Therapie durch Zuführung von Beatmungsgeräten usw. vorzubereiten.

\section{Welche Informationen muss ein Notfallplan enthalten?}

Das gehäufte Auftreten von VHF ist in Mitteleuropa unwahrscheinlich, aber im Zusammenhang mit einem bioterroristischen Angriff nicht auszuschließen. Auch die SARS-Epidemie hat gezeigt, wie manche Krankenhäuser kurzfristig von einer großen Anzahl infektiöser Patienten betroffen sein können [5, 6].

Im Notfallplan ist grundsätzlich zu unterscheiden, ob ein Einzelpatient oder mehrere Patienten zu versorgen sind. Eine weitere Unterteilung bietet sich

Tab. 9 Allgemeines Vorgehen bei Einzelpatienten (Virusbedingtes Hämorrhagisches Fieber (VHF), Lungenpest, Pocken, schwere pandemische Influenza-Infektion, Severe Acute Respiratory Syndrome (SARS)

\begin{tabular}{ll} 
Maßnahme & Beispiel \\
\hline Im Bereich der & Schutz des Personals mit Infektionsschutzsets \\
Notaufnahme/ & Isolierung des Patienten in einem definierten \\
Rettungsstelle & Raum \\
& Sicherung der Vitalfunktionen \\
& Nach Möglichkeit unverzüglicher Transport des \\
& Patienten in das Kompetenzzentrum; falls dies \\
& nicht möglich ist, Transport in die besondere \\
& Behandlungszone \\
& - Isolierung von direkten Kontaktpersonen in ei- \\
& nem definierten Raum \\
& Ggf. Verschluss der Notaufnahme/Rettungs- \\
& stelle und Einrichtung eines Ausweichbetriebs \\
& in einem definierten Bereich \\
- & Schutz des Personals mit Infektionsschutzsets \\
- & Geeigneten Raum (möglichst mit Schleuse) \\
Im Bereich der & räumen und für den Patienten vorbereiten \\
besonderen Behand- & (Zugang möglichst von außen) \\
lungszone (sofern kein & Regeln der Klimaanlage sofern erforderlich \\
Transport zum & Festlegung der Desinfektionsmaßnahmen \\
Kompetenzzentrum & Festlegung des Umgangs mit Wäsche und \\
möglich ist) & Abfall usw. \\
& - Adresse eines kompetenten Infektiologen \\
(innerhalb oder außerhalb) \\
Kontaktadressen/ & - Gesundheitsamt (Meldung) \\
Telefonnummern & Nächstgelegenes Kompetenzzentrum \\
& (Verlegung, Beratung) \\
& - Labore: Diensthabender Mikrobiologe/Virologe \\
& Technische Leitwarte (Klimaanlage) \\
\hline &
\end{tabular}


Abb. 1 Die fünf Kompetenzzentren in Deutschland

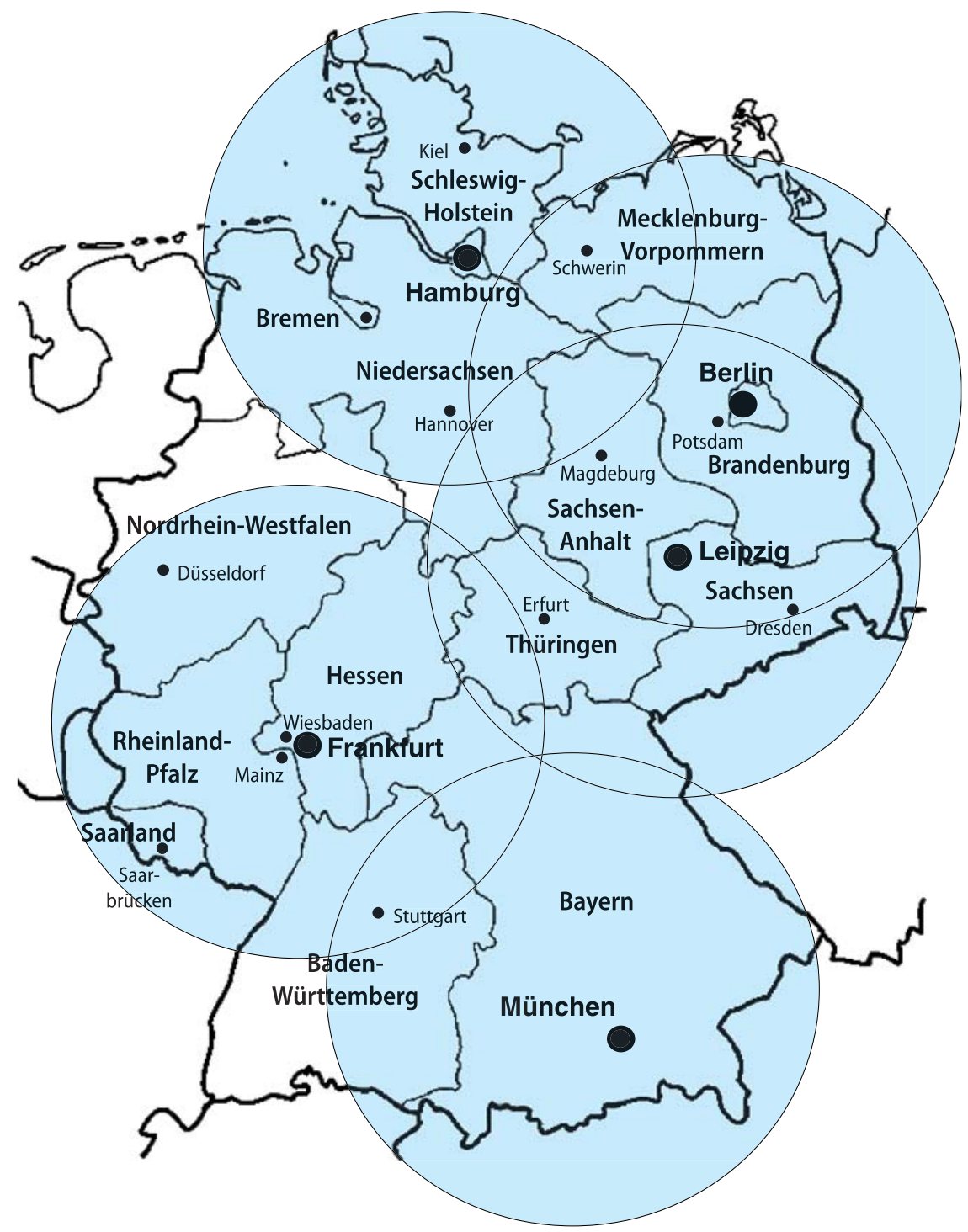

nicht an, weil der organisatorische Aufwand im Vordergrund steht und die Patientenzahl in den Hintergrund tritt. Im Einzelnen sind die in Tab. 9 und 10 aufgelisteten Aspekte zu beachten.

Die folgende Checkliste (Tab. 11) soll zur Orientierung bei der Erstellung einer Alarmanweisung dienen.

Es genügt nicht, entsprechende Infektionsschutzsets für das Personal bereitzuhalten; der richtige Umgang mit der Schutzkleidung muss im Vorfeld durchgesprochen und geübt werden (Tab. 12 nach [3]).

\section{Desinfektionsmaßnahmen und Abfallentsorgung}

Wenn eine hochkontagiöse Infektion vermutet wird, sollen möglichst Einwegmaterialien verwendet wer- den. Im übrigen sollen Instrumente, Geschirr, Wäsche und Textilien innerhalb des Raumes desinfiziert werden, in dem sie anfallen [7]. Im Falle einer Virusinfektion ist eine tägliche Scheuerwischdesinfektion der patientennahen Flächen mit einem gegen Viren wirksamen Mittel der Liste der vom RKI geprüften und anerkannten Desinfektionsmittel und -verfahren durchzuführen (Wirkungsbereich $\mathrm{AB}$ ). Für Matratzen werden wischdesinfizierbare Überzüge empfohlen. Erregerhaltiges Material und Abfälle, die mit erregerhaltigem Material kontaminiert sein könnten, sind ebenfalls vor Verlassen des Raumes zu desinfizieren. Anschließend werden sie entsprechend den krankenhauseigenen Richtlinien für infektiöse Abfälle entsorgt. Die Schlussdesinfektion des Raumes erfolgt durch Verdampfen oder Vernebeln von Formaldehyd und anschließende Scheuerwischdesinfekti- 
Tab. 10 Vorgehen bei mehreren Patienten/Massenanfall. Zur Sicherung der allgemeinen Funktionsfähigkeit des Krankenhauses ist für die Übernahme der Patienten eine besondere Aufnahmezone festzulegen, für die Isolierung und Behandlung eine besondere Behandlungszone und für die externe Verlegung von Infektionspatienten eine besondere Verlegezone

\begin{tabular}{|c|c|}
\hline Maßnahme & Beispiel \\
\hline $\begin{array}{l}\text { Besondere } \\
\text { Aufnahmezone }\end{array}$ & $\begin{array}{l}\text { - Festlegung der Anfahrt der Rettungsmittel mit } \\
\text { Übernahmebereich } \\
\text { - Sicherung gegen unbefugtes Betreten von } \\
\text { innerhalb und außerhalb des Gebäudes } \\
\text { - Erfassung der Patienten durch den Aufnahme- } \\
\text { dienst } \\
\text { - Sichtung durch die erfahrensten Fachärzte für } \\
\text { Innere Medizin und Anästhesiologie }\end{array}$ \\
\hline $\begin{array}{l}\text { Besondere } \\
\text { Behandlungszone }\end{array}$ & $\begin{array}{l}\text { Räumung des Bereichs von allen verlegefähigen } \\
\text { Patienten } \\
\text { - Festlegung eines Bereiches für die Erstversor- } \\
\text { gung zur Sicherung der Vitalfunktionen } \\
\text { Festlegung eines Bereiches für die weitere } \\
\text { intensivmedizinische Behandlung } \\
\text { Festlegung eines Bereichs für die Betreuung von } \\
\text { nicht sofort behandlungsbedürftigen Patienten } \\
\text { - Festlegung eines Bereichs für die Betreuung } \\
\text { von hoffnungslosen Patienten } \\
\text { Personalwechsel ausschließlich über definierte } \\
\text { Schleusen (auf das Minimum reduzieren) } \\
\text { Festlegung eines Quarantänebereichs der } \\
\text { Mitarbeiter } \\
\text { - Materialversorgung über definierte Schleusen } \\
\text { Betreuung der Angehörigen vorbereiten }\end{array}$ \\
\hline Besondere Verlegezone & $\begin{array}{l}\text { Verlegungswege definieren und Verantwortlich- } \\
\text { keit festlegen }\end{array}$ \\
\hline Personal & $\begin{array}{l}\text { Die Patienten werden durch anwesende und } \\
\text { ggf. nachalarmierte Mitarbeiter der folgenden } \\
\text { Abteilungen versorgt } \\
\text { Zur kontinuierlichen Versorgung von Notfall- } \\
\text { patienten sind Behandlungsteams - in der } \\
\text { Regel bestehend aus Internist, Anästhesist } \\
\text { (ersatzweise Arzt mit Zusatzbezeichnung „Not- } \\
\text { fallmedizin“) und Fachpflegekraft Anästhesiolo- } \\
\text { gie/Intensivmedizin (ersatzweise sonstige } \\
\text { Pflegekraft) - zu bilden }\end{array}$ \\
\hline Material & $\begin{array}{l}\text { Infektionsschutzsets für die Erstausstattung der } \\
\text { Mitarbeiter bereitstellen } \\
\text { - Weitere Versorgung organisieren }\end{array}$ \\
\hline
\end{tabular}

on mit Mitteln und Verfahren der RKI-Liste durch einen Desinfektor.

Bei SARS und Verdacht auf schwere pandemische Influenza sollen alle Medizinprodukte mit direktem Kontakt zum Patienten patientenbezogen verwendet und nach Gebrauch desinfiziert werden. Hier muss die Desinfektion nicht unbedingt vor Ort erfolgen; sie kann bei Transport in geschlossenen Behältern auch in der zentralen Aufbereitung vorgenommen werden. Dort sollen vorzugsweise thermische Desinfektionsverfahren eingesetzt werden. Auch Geschirr kann in einem geschlossenen Behältnis zur Spülmaschine transportiert werden und wird dort maschi- nell aufbereitet $\left(>60^{\circ} \mathrm{C}\right)$. Wäsche wird einem desinfizierenden Waschverfahren zugeführt. Eine Verneblung von Formaldehyd zur Schlussdesinfektion ist nicht erforderlich (RKI-Empfehlungen, Stand Februar 2006). Die Abfallentsorgung erfolgt nach Abfallschlüssel EAK 180104 gemäß LAGA-Richtlinie (LAGA = Landesarbeitsgemeinschaft Abfall).

\section{Überwachung exponierter Personen}

Bei VHF wurde die Mehrzahl der Sekundärfälle bei Personen festgestellt, die Kranke betreut hatten. Das Übertragungsrisiko ist insbesondere im fortgeschrittenen Krankheitsstadium erhöht, wahrscheinlich als Folge der in dieser Phase auftretenden Diarrhoe sowie von Erbrechen und Blutungen. Obwohl das Virus bereits einige Tage vor Krankheitsausbruch nachweisbar ist, wurden bislang keine Übertragungen durch Kontakte im asymptomatischen präklinischen Stadium beobachtet. Abhängig vom Ausmaß des Kontaktes und der Sicherung der Diagnose des VHF beim Indexfall kann es sinnvoll sein, für Personen mit erhöhtem Expositionsrisiko die gleichen Schutzvorkehrungen inklusive Isolierung $\mathrm{zu}$ treffen wie für den Indexpatienten [8]. Im Allgemeinen wird folgende Kategorisierung der Kontaktpatienten vorgenommen (Tab. 13).

Entsprechend $\$ 30$ des Infektionsschutzgesetzes (IfSG) kann bei Kontaktpersonen eine Absonderung durch die zuständige Behörde angeordnet werden. Das Gesundheitsamt hat die jeweils entsprechenden Maßnahmen festzulegen. Bei Personen mit hohem Risiko eines Kontaktes zu einem VHF-Patienten erfolgt eine Überwachung der Körpertemperatur sowie eventuell eine medikamentöse Prophylaxe mit Ribavirin. Bei niedrigem Risiko ist ebenfalls die regelmäßige Überwachung der Körpertemperatur empfohlen. Kein Risiko ist für Personen gegeben, die im selben Flugzeug wie der Indexpatient gereist sind, die ausschließlich Sichtkontakt hatten sowie für Pflege- und Laborpersonal unter Einhaltung der beschrieben Schutzmaßnahmen.

\section{Fazit}

Die Übertragungswege und die Kontagiösität der verschiedenen Infektionserreger sind unterschiedlich. Beim Auftreten des Verdachtes auf eine allgemeingefährliche Infektionskrankheit ist meistens nicht klar, um welchen Erreger es sich handeln könnte. Deshalb müssen die Präventionsmaßnahmen von dem allgemeinen Verständnis der Übertragung von Infektionskrankheiten und dem maximalen Schutz für Mit- 
Tab. 11 Checkliste 2 - Versorgung eines Einzelpatienten mit allgemeingefährlicher Infektionskrankheit in der Notaufnahme/Rettungsstelle (am Beispiel der MHH)

\begin{tabular}{|c|c|c|}
\hline Problem & Maßnahmen/Beispiel & Ansprechpartner/Kontakt \\
\hline Zuständiger Arzt & Information des Arztes vom Dienst & Telefonnummer \\
\hline Zuständiges Gesundheitsamt & Information des Gesundheitsamtes & $\begin{array}{l}\text { Telefonnummer in der Dienstzeit und außerhalb der } \\
\text { Dienstzeit }\end{array}$ \\
\hline Zuständiges Kompetenzzentrum & Bernhard-Nocht-Institut informieren & (040) 428180 (24-stündige Rufbereitschaft) \\
\hline $\begin{array}{l}\text { Zuständiges mikrobiologisches } \\
\text { Labor }\end{array}$ & $\begin{array}{l}\text { Geeignetes Labor informieren, } \\
\text { Absprache mit diensthabendem Virologen und Mikrobiologen }\end{array}$ & $\begin{array}{l}\text { Bernhard-Nocht-Institut; } \\
\text { Telefonnummern diensthabender Mikrobiologe/ } \\
\text { Virologe: }\end{array}$ \\
\hline $\begin{array}{l}\text { Verbleib von Patienten/ } \\
\text { Angehörigen }\end{array}$ & $\begin{array}{l}\text { Belassen der betroffenen Person am Aufnahmeort und Absonderung } \\
\text { von Nichtbetroffenen } \\
\text { Patient und Kontaktpersonen 1. Grades in getrennten Zimmern } \\
\text { (möglichst mit Schleusenfunktion) isolieren }\end{array}$ & Räume festlegen \\
\hline Bautechnik-Maßnahmen & $\begin{array}{l}\text { Klimaanlage auf Unterdruck umschalten bzw. abkoppeln } \\
\text { Fenster und Türen geschlossen halten }\end{array}$ & Telefonnummer technische Leitwarte \\
\hline $\begin{array}{l}\text { Alternative Notfallaufnahme } \\
\text { einrichten }\end{array}$ & $\begin{array}{l}\text { Räumung des vorgesehenen Bereichs } \\
\text { Personelle und materielle Ausstattung } \\
\text { Verständigung der Rettungsleitstelle über diesen Ortswechsel }\end{array}$ & Telefonnummer \\
\hline Ordnungsbehörden & Umgehende provisorische Abriegelung des kontaminierten Bereiches & Telefonnummer Polizei \\
\hline Rekrutierung Personal & $\begin{array}{l}\text { Möglichst nur geschultes Personal einsetzen, es darf nicht mehr für } \\
\text { die Versorgung anderer Patienten eingesetzt werden (Anordnung } \\
\text { Oberarzt) }\end{array}$ & $\begin{array}{l}\text { Telefonnummern Oberarzt, } \\
\text { Pflegedienstleitung }\end{array}$ \\
\hline Personalschutz & $\begin{array}{l}\text { Vor jedem Patientenkontakt sowie bei Betreten des Zimmers } \\
\text { Anlegen von Mund-Nasenschutz, Schutzanzug oder -kittel, } \\
\text { Überschuhen, Einmalhandschuhen, Kopfhaube und Schutzbrille } \\
\text { Hygienische Händedesinfektion vor und nach Tragen der Handschuhe } \\
\text { Postexpositionelle Vakzinierung des Personals (bei Pockenverdacht) }\end{array}$ & $\begin{array}{l}\text { Notfall-Sets in Notaufnahme deponieren } \\
\text { Telefonnummmer zur Bestellung von weiteren Sets } \\
\text { Nach Absprache mit dem Landesgesundheitsamt }\end{array}$ \\
\hline Transport & $\begin{array}{l}\text { Transport in einem speziell eingerichteten Fahrzeug } \\
\text { Patient mit Mund-Nasenschutz, Haube, Schutzkittel }\end{array}$ & Rettungsleitstelle \\
\hline Weiterverlegung & Wenn Verlegung in das Haus (Infektionsstation) erforderlich & Telefonnummer diensthabender Internist \\
\hline Desinfektion & $\begin{array}{l}\text { Flächen: Desinfektionsmittel mit Konzentration und Einwirkungszeit } \\
\text { festlegen } \\
\text { Schlussdesinfektion als Raumdesinfektion durch Verdampfen und Ver- } \\
\text { nebeln von Formaldehyd durch einen Desinfektor mit anschließender } \\
\text { Scheuerwischdesinfektion sämtlicher zugänglicher Flächen und Gegen- } \\
\text { stände } \\
\text { Instrumente: Abwurf in Behälter mit definiertem Desinfektionsmittel }\end{array}$ & $\begin{array}{l}\text { Telefonnummer Krankenhaushygiene } \\
\text { bzw. über diensthabenden Mikrobiologen, } \\
\text { Piepser-Nr. der Desinfektoren }\end{array}$ \\
\hline Wäsche & $\begin{array}{l}\text { Personalwäsche: C-Müll = schwarze Tonne } \\
\text { Patientenwäsche: C-Müll = schwarze Tonne }\end{array}$ & \\
\hline
\end{tabular}

Tab. 12 Arbeitsschritte beim Betreten und Verlassen eines Isolierzimmers (nach [3])

\begin{tabular}{ll}
\hline Arbeitsschritt & $\begin{array}{l}\text { Reihenfolge der Maßnahmen } \\
\text { Betreten der Schleuse }\end{array}$ \\
& $\begin{array}{l}\text { Nach hygienischer Händedesinfektion Overall oder } \\
\text { Überschürze sowie Überschuhe anziehen, Gesichts- } \\
\text { maske anlegen, Schutzbrille aufsetzen, Handschuhe } \\
\text { anziehen, Zimmer betreten }\end{array}$ \\
Zimmer verlassen & $\begin{array}{l}\text { Overall oder Überschürze sowie Überschuhe } \\
\text { ausziehen, Gesichtsmaske und Schutzbrille ablegen, } \\
\text { Handschuhe ausziehen, gesamtes Einmalmaterial } \\
\text { entsorgen, hygienische Händedesinfektion }\end{array}$
\end{tabular}

Tab. 13 Kategorisierung von Kontaktpersonen (bei VHF und Pocken)

\section{Kategorie Beschreibung der Exposition}

la Hohes Risiko: Personen, die direkten/invasiven Hautkontakt mit Blut anderen Körperflüssigkeiten von erkrankten Personen hatten

Ib Erhöhtes Risiko: Personen, die auf intakter Haut Kontakt mit Blut oder Körperflüssigkeiten oder Kontakt mit Aerosol hatten

II Mäßiges Risiko: Personen, die Kontakt zu erkrankten Personen hatten und die mit Blut oder anderen Körperflüssigkeiten kontaminiert gewesen sein könnten

III Geringes Risiko: Personen, die Kontakte zu Erkrankten hatten, bei denen aber kein Kontakt mit Blut oder Körperflüssigkeiten bestand bzw. medizinisches Personal, das intakte Schutzanzüge und Respiratoren getragen hat 
Abb. 2 Entscheidungsfindung vom Verdachtsmoment zum Verdachtsfall

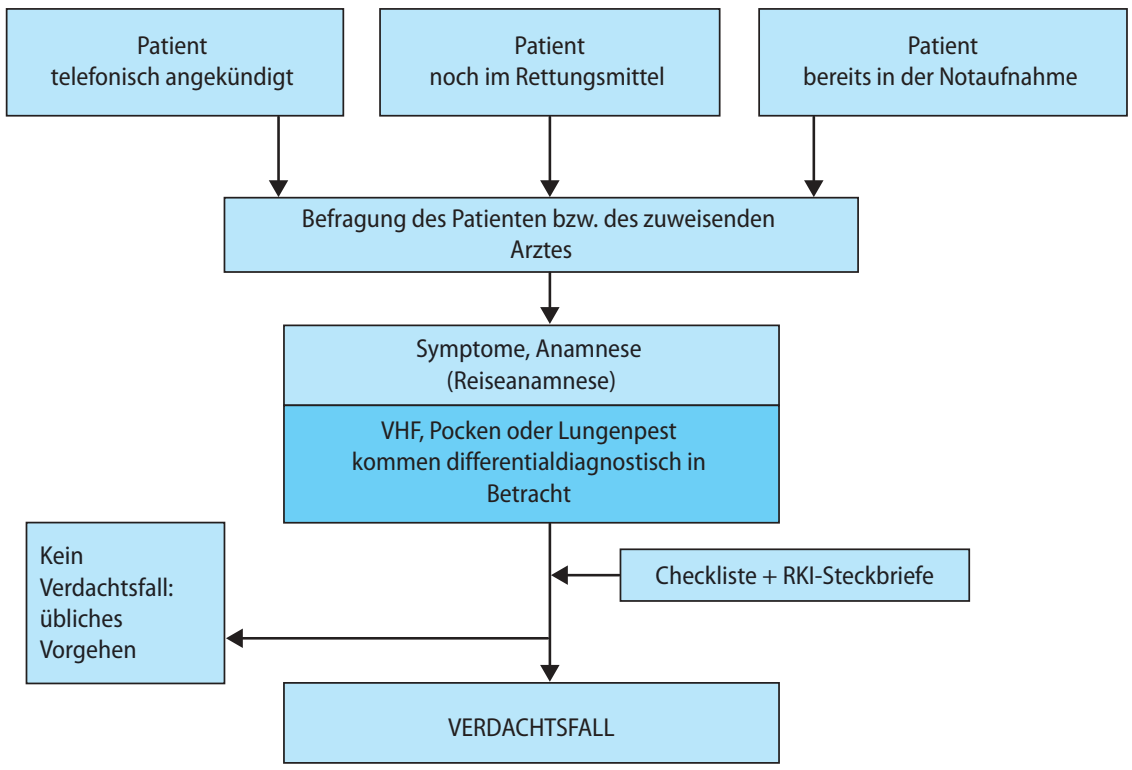

Abb. 3 Management eines telefonisch angekündigten Patienten, eines bereits mit dem Krankentransport vorgefahrenen Patienten sowie eines Patienten, der bereits in der Notaufnahme ist. Wenn möglich sollte bei entsprechendem Verdacht von vorn herein vermieden werden, dass der Patient das Krankenhaus betritt

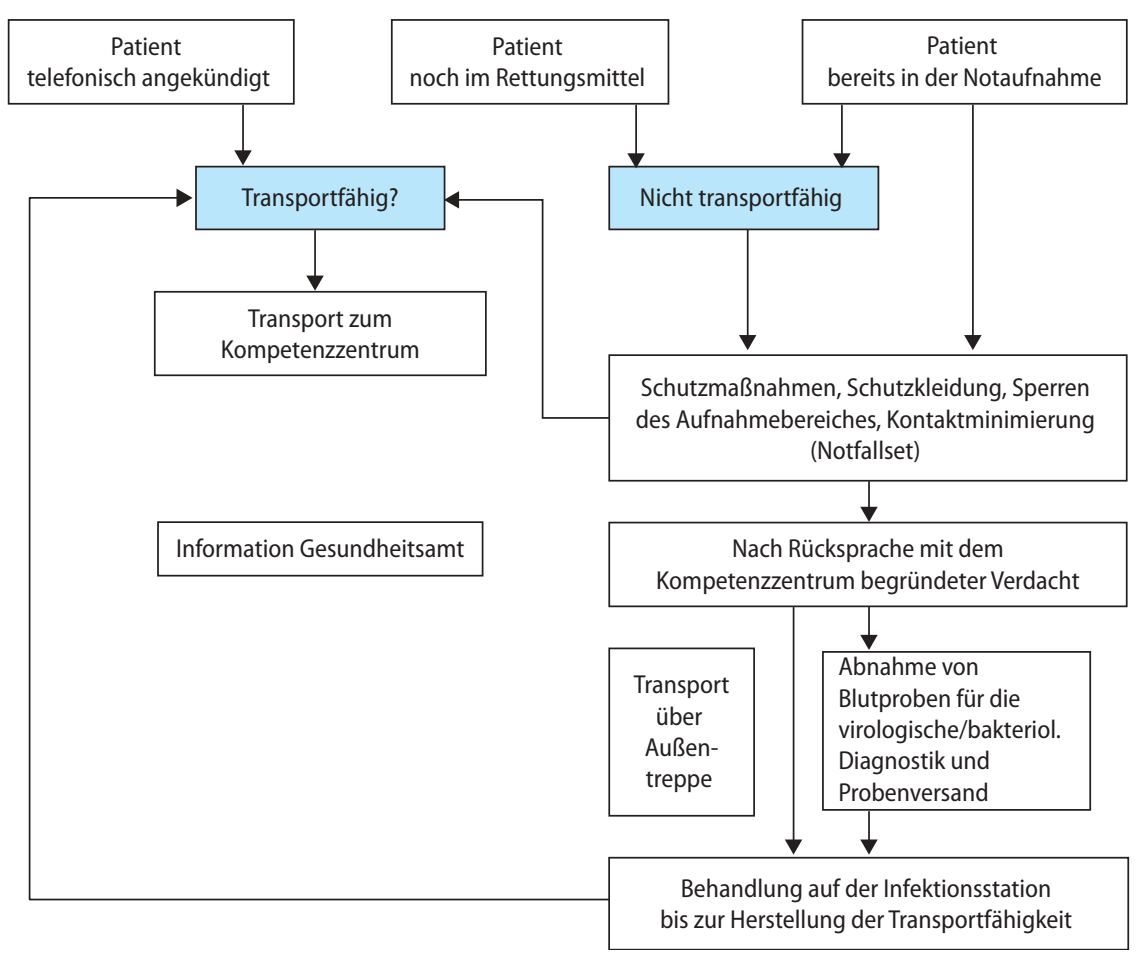

patienten und Personal ausgehen. Im weiteren Verlauf der Behandlung müssen die Maßnahmen an die konkreten Bedingungen des jeweiligen Erregers angepasst werden.

\section{Wichtige Internetadressen}

Homepage RKI: $w w w . r k i . d e$

Homepage CDC: $w w w . c d c . g o v$

Homepage WHO: www.who.int 


\section{Literatur}

1. Papineni R, Rosenthal F (1997) The size distribution of droplets in the exhaled breath of healthy human subjects. J Aerosol Med 10:105-116

2. Siegel J, Strausbaugh L, Jackson M, Rhinehart E, Chiarello L (2004) Draft HICPAC Guideline for isolation precautions: Preventing transmission of infectious agents in health care settings. www.cdc.gov

3. Hugonnet S, Sax H, Chappuis F, Mühlemann K, Francoli P, Raeber P-A, Hatz C, Widmer A, Siegl G, die Mitglieder der Swiss-NOSO und das Scientific Advisory Board der Schweizer Gesellschaften für Infektiologie $M$, Tropenmedizin und Sozial- und Präventivmedizin (2002) Schutzmaßnahmen bei Patienten mit Verdacht auf virales hämorrhagisches Fieber: Empfehlungen für die Schweiz. Swiss-Noso 9:1-13
4. Fock R, Koch U, Finke E-J, Niedrig M, Wirtz A, Peters M et al (2000) Schutz vor lebensbedrohenden importierten Infektionskrankheiten. Bundesgesundheitsbl Gesundheitsforsch Gesundheitsschutz, 43

5. Lee $\mathrm{N}$, Hui $\mathrm{D}, \mathrm{Wu} \mathrm{A}$, Chan $\mathrm{P}$, Cameron P, Joynt G (2003) A major outbreak of severe acute respiratory syndrome in Hong Kong. N Eng J Med 348:19861994

6. McDonald L, Simor A, Su I-J, Maloney S, Ofner M, Chen K-T (2004) SARS in healthcare facilities Toronto and Taiwan. Emerg Inf Dis 10:777-781
7. Fock R, Wirtz A, Peters M, Finke E-J, Koch U, Scholz D, Niedrig M, Bußmann H, Fell G, Bergmann H (1999) Management und Kontrolle lebensbedrohender hochkontagiöser Infektionskrankheiten. Bundesgesundheitsbl Gesundheitsforsch Gesundheitsschutz 42: 389-401

8. Ruef C, Raeber P-A, BAG, und das Redaktionskomitee von Swiss-NOSO (1996) Vorsichtsmaßnahmen im Spital bei vermuteten oder gesicherten Fällen mit viraöem hämorrhagischem Fieber. Swiss-NOSO 3 